Research Paper

\title{
Numerical simulation of the fracture process in ceramic FPD frameworks caused by oblique loading
}

\author{
Wen Kou , Jiyan Qiao $^{b}$, Li Chen ${ }^{b}$, Yansheng Ding ${ }^{b}$, Göran Sjögren ${ }^{a, *}$ \\ ${ }^{a}$ Dental Materials Science, Faculty of Medicine, Umeå University, SE-90187, Umeå, Sweden \\ bInstitute of Mechanics, Chinese Academy of Sciences, CN-100190, Beijing, China
}

\section{A R T I C L E I N F O}

\section{Article history:}

Received 28 March 2015

Received in revised form

13 June 2015

Accepted 15 June 2015

Available online 24 June 2015

Keywords:

Finite element analysis

Fixed partial denture

Fracture process

Numerical simulation

Oblique loading

\begin{abstract}
A B S T R A C T
Using a newly developed three-dimensional (3D) numerical modeling code, an analysis was performed of the fracture behavior in a three-unit ceramic-based fixed partial denture (FPD) framework subjected to oblique loading. All the materials in the study were treated heterogeneously; Weibull's distribution law was applied to the description of the heterogeneity. The Mohr-Coulomb failure criterion with tensile strength cut-off was utilized in judging whether the material was in an elastic or failed state. The simulated loading area was placed either on the buccal or the lingual cusp of a premolar-shaped pontic with the loading direction at $30^{\circ}, 45^{\circ}, 60^{\circ}, 75^{\circ}$ or $90^{\circ}$ angles to the occlusal surface. The stress distribution, fracture initiation and propagation in the framework during the loading and fracture process were analyzed. This numerical simulation allowed the cause of the framework fracture to be identified as tensile stress failure. The decisive fracture was initiated in the gingival embrasure of the pontic, regardless of whether the buccal or lingual cusp of the pontic was loaded. The stress distribution and fracture propagation process of the framework could be followed step by step from beginning to end. The bearing capacity and the rigidity of the framework vary with the loading position and direction. The framework loaded with $90^{\circ}$ towards the occlusal surface has the highest bearing capacity and the greatest rigidity. The framework loaded with $30^{\circ}$ towards the occlusal surface has the least rigidity indicating that oblique loading has a major impact on the fracture of ceramic frameworks.
\end{abstract}

(c) 2015 Elsevier Ltd. All rights reserved.

\section{Introduction}

Ceramics are among the most inert biomaterials known. Their advantages include high biocompatibility (Depprich et al., 2008), good esthetic appearance (Ghazal et al., 2008), low solubility and low thermal conductivity (Chai et al., 2007). Among the crucial problems for ceramics, however, are low fracture resistance and brittleness and, consequently, no or little apparent plastic deformation can occur before fracture (Munz and Fett, 1999). Due to the limitation of their mechanical properties ceramics have mostly been used for crowns and short-span fixed partial dentures (FPDs) in the anterior region (Sorensen et al., 1998;

\footnotetext{
*Corresponding author. Tel: +46907856132.

E-mail address: goran.sjogren@umu.se (G. Sjögren).
} 
Cehreli et al., 2011). In the recent decades more advanced ceramic materials, such as oxide-ceramics, have been introduced onto the market leading to a wider application of ceramic material for crowns and FPDs both in the anterior and posterior part in the oral cavity (Håff et al., 2015).

Although the mechanical properties of ceramics have been improved, once a fracture is initiated in a ceramic FPD (c-FPD) framework it still often leads to a total failure (Cehreli et al., 2011). A better understanding of the fracture mechanism in ceramics could lead to the avoidance of fractures and one possible way of analyzing fracture processes in ceramics is to use mathematical methods (Holberg et al., 2013). Numerical simulation is an example of a mathematical solution able to solve problems in an approximate manner and deliver satisfactory results (Lee and Lim, 2013). One commonly used numerical simulation method is the finite element method (FEM), i.e. a numerical technique for finding approximate solutions for partial differential equations or integral equations (Holberg et al., 2013). The vast development in computer technology means that the calculation speed has increased, leading to extended applications of the numerical method (Holberg et al., 2013; Lee and Lim, 2013; Papanicolaou et al., 2015). In a previous paper, a three-dimensional (3D) numerical simulation was applied to studying the fracture process in a three-unit dental c-FPD framework under perpendicular mechanical loading towards the occlusal surface (Kou et al., 2011). The fracture in the previous numerical simulation test largely correlated with the patterns of the results in a corresponding laboratory test (Sundh et al., 2005).

In the oral cavity, however, the loading is seldom perpendicular to the occlusal surface but instead is often oblique. In a survey of the literature in the database (PubMed), no article was found that addresses the effect of various oblique loading on stress distribution and fracture propagation in cFPDs frameworks. The aim of the present study, therefore, was to evaluate the effect of oblique loading on the fracture process in a three-unit c-FPD framework using a numerical simulation technique.

\section{Materials and methods}

All the materials used in the present simulation were assumed to be mesoscopically heterogeneous. The Weibull distribution law (Weibull, 1951) was utilized to assign values of the element parameters to give the mesoscopical elements their varying mechanical properties.

The Weibull distribution law is described by the following formula:

$\varphi(\sigma)=\left\{\begin{array}{c}\frac{m}{\sigma_{0}}\left(\frac{\sigma}{\sigma_{0}}\right)^{m-1} \exp \left[-\left(\frac{\sigma}{\sigma_{0}}\right)^{m}\right], \sigma \geq 0 \\ 0, \sigma<0\end{array}\right.$

where $\varphi(\sigma)$ is the probability density function of $\sigma, \sigma$ is the element parameter which can be tensile strength or compressive strength or Young's modulus of the element, $\sigma_{0}$ is the mean value of the element parameter and $m$ is the Weibull modulus. A larger $m$ implies a more homogeneous material. The Monte Carlo method was applied to achieve the specific heterogeneity distribution in 3D physical space after the generation of finite elements (Liu, 2004).

The relationship of elasticity to stress and strain of the element was observed using Hooke's law. The Mohr-Coulomb strength criterion with tensile strength cut-off was applied in judging whether the element was in an elastic or failed state.

The Mohr-Coulomb strength criterion is expressed as the following formula:

$\left|\tau_{\mathrm{P}}\right|+\sigma_{\mathrm{P}} \tan \phi-\tau_{\mathrm{S}} \geq 0$

and the tensile strength cut-off is

$\sigma_{1} \geq \sigma_{\mathrm{t}}$

where $\tau_{p}$ is the shear stress and $\sigma_{p}$ is the normal stress for one surface element sectioned through the point examined. In the present study tensile stress is assumed to be positive and, therefore, $\sigma_{1}$ is the maximum principal stress at that point. $\phi$ and $\tau_{\mathrm{s}}$ are the friction angle and the shear strength of the element, respectively and $\sigma_{t}$ is the uniaxial tensile strength of the element. The element will be in a state of failure if Eq. (2) and/or Eq. (3) are/is valid. Once the element fails the elastic modulus will degrade by an empirical factor of $R$.

The geometric boundary of the three-unit ceramic-based framework with abutments is shown in Fig. $1 \mathrm{~d}$ and e. The displacement load was applied on the occlusal surface of the simulated framework (Fig. 1b). The relationship mentioned above, together with the boundary conditions, describes a complete mechanical problem for obtaining the deformation and fracture of the framework under loading and in order to solve this problem the numerical methods were applied.

The numerical simulation code used in the present study was developed at the Institute of Mechanics, Chinese Academy of Sciences in Beijing, China. It is based on GiD (CIMNE, Barcelona, Spain), a 3D graphic pre-and post-processor for computer simulation and Finite Element Program Generator (FEPG, Fegensoft, Beijing, China), which is a platform for generating finite element modeling codes. Since the geometric shape of the framework was designed by a computer-aided design (CAD) program, representative nodes were selected from the CAD data (Fig. 1d) and the coordinators of the nodes were entered into GiD to generate finite elements for the solid model of the framework. A total of 302,200 tetrahedral elements without mid-side nodes were generated. The location of the loading areas was simulated either on the buccal or the lingual cusp of the premolar-shaped pontic, with the loading direction at $30^{\circ}, 45^{\circ}, 60^{\circ}, 75^{\circ}$ or $90^{\circ}$ angles to the occlusal surface. Displacement of the loading area was then applied $0.002 \mathrm{~mm}$ step by step.

Table 1 lists the mechanical properties of the materials used. The three-unit c-FPD framework and abutments were simulated as yttria-tetragonal zirconia polycrystal (Y-TZP) ceramics, with soft layers placed under the abutments (Fig. 1e). Both the Y-TZP ceramic and the soft layers were assumed to be heterogeneous materials. Deformations of the abutments and the soft layers were also simulated. The displacement in the normal direction at the lowest surfaces of the soft layers was zero. The normal stress perpendicular and shear stress parallel to the boundary surface of the other parts of the framework were zero.

The progressive failure of each element is described by a variable termed as 'flag'. The definition of the flag is $N_{f} / N$, where $N$ is the total number of the element nodes and $N_{f}$ is the number 


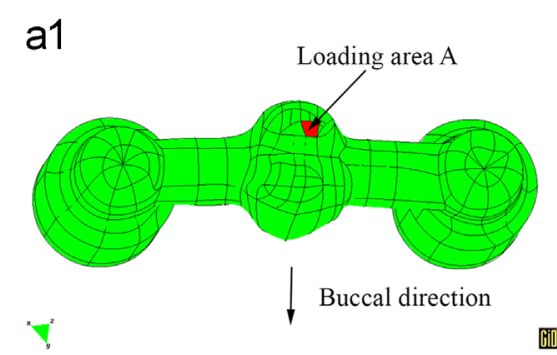

b1

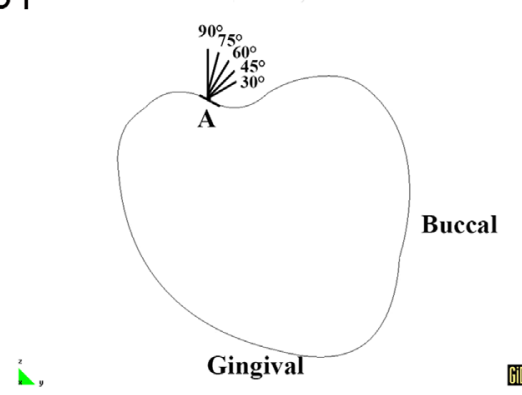

C

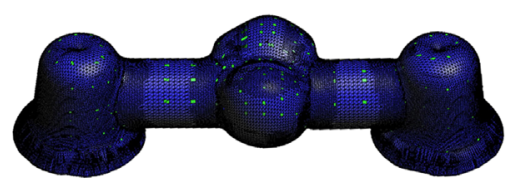

a2

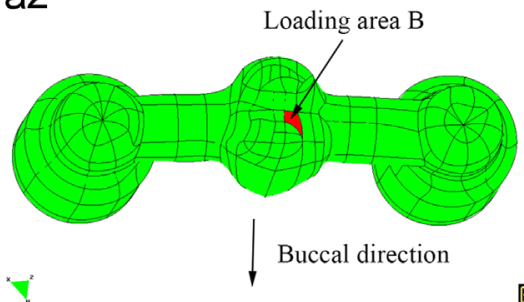

四

b2

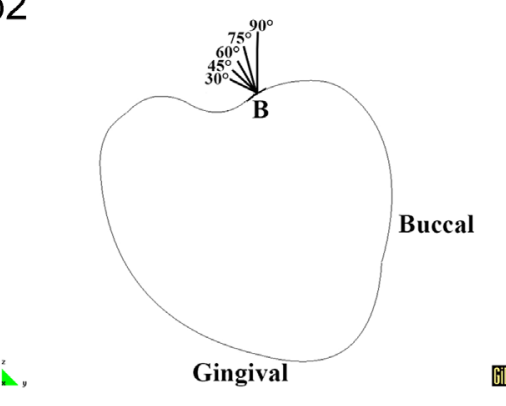

d

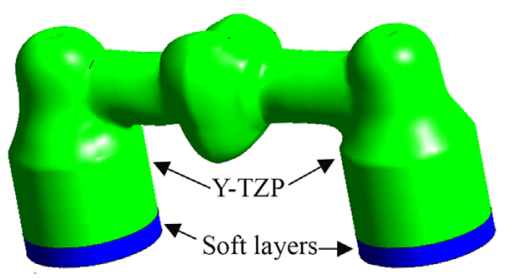

Fig. 1 - (a) Occlusal view of the geometric model of the framework for the numerical simulation. The loading areas are marked with red color either on the lingual (a1) or the buccal cusp (a2) of the pontic-shaped premolar. (b) The simulated loading directions were placed at $30^{\circ}, 45^{\circ}, 60^{\circ}, 75^{\circ}$ or $90^{\circ}$ angles to the occlusal surface. Loading area A denotes the loading on the lingual cusp (b1) and B denotes the loading on the buccal cusp (b2). (c) Lingual view of the three-unit framework from the CADfile. The color green marks the location of the selected nodes on the geometric model. d) Buccal view of the three-unit framework. The green area is simulated as Y-TZP and the blue areas as the soft layers. (For interpretation of the references to color in this figure legend, the reader is referred to the web version of this article.)

Table 1 - The mechanical properties of the materials studied in the present study.

\begin{tabular}{lll} 
& Y-TZP & Soft layer \\
\hline Young's modulus (GPa) & $220^{\mathrm{a}}$ & 22 \\
Tensile strength (MPa) & $840^{\mathrm{a}}$ & $84 \times 10^{4}$ \\
Uniaxial compressive strength (MPa) & $3500^{\mathrm{b}}$ & $22 \times 10^{4}$ \\
Poisson's ratio & $0.25^{\mathrm{b}}$ & 0.26 \\
Weibull modulus & $10^{\mathrm{c}}$ & 10 \\
\hline${ }^{\mathrm{a}}$ Guazzato et al. (2004). & & \\
${ }^{\mathrm{b}}$ Information from the manufacturer. & & \\
${ }^{\mathrm{c}}$ Guazzato et al. (2005). & & \\
\hline
\end{tabular}

which failed. An element had completely failed when flag=1 and was without any damage when flag $=0$. An element was partly damaged when $0<$ flag $<1$ and the increase in flag from 0 to 1 describes the progressive failure of the element.

Finally, the stress distribution, fracture initiation and propagation of the three-unit c-FPD framework were analyzed. The bearing capacity and the rigidity of the framework with varying degrees of oblique loading were studied further.

\section{Results \\ 3.1. Fracture mechanism}

The stress distribution and fracture propagation of a three-unit c-FPD framework could be followed step by step from the beginning of the loading of the framework to the culmination of the total framework fracture. The concentration of the tensile and compressive stress in the framework varies throughout the simulated mechanical loading process (Fig. 2). At the moment before the framework fractured, there was high tensile stress concentration in the gingival embrasure of the framework and high compressive stress concentration in the occlusal area (Fig. 2). The first fracture detected in the framework was in the occlusal area connected to the loaded area. However, this was not the main reason for the total framework fracture; that was 
a

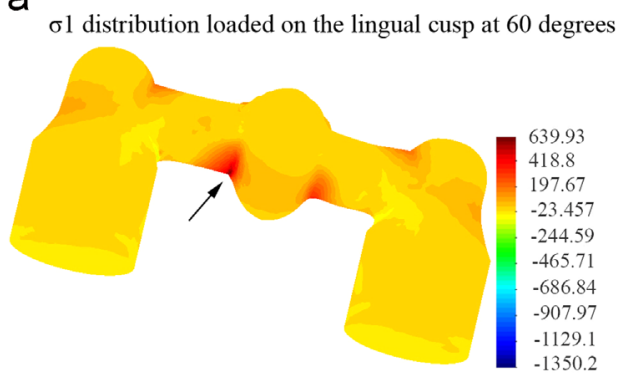

b

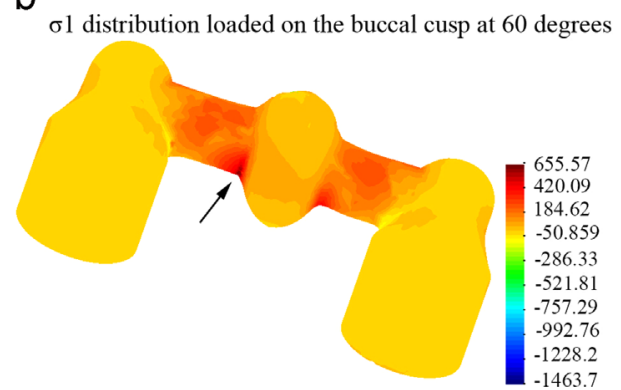

Fig. 2 - Images of simulated maximum principal stress $\left(\sigma_{1}\right)$ distributions in the framework with loading on the lingual (a) and buccal (b) cusp with loading at $60^{\circ}$ angle to the occlusal surface before the framework fractures. The rainbow colored marker indicates the stress value (MPa). The bluest color indicates the lowest stress and the reddest color indicates the highest stress. The highest tensile stress could be observed in the gingival embrasure and is indicated with black arrows. (For interpretation of the references to color in this figure legend, the reader is referred to the web version of this article.)

tensile stress and the crucial initiation area of the failure was in the gingival embrasure of the framework, regardless of whether the buccal or the lingual cusp was loaded (Fig. 2).

\subsection{Effect of direction of the loading on the displacement and load at fracture}

The load and displacement curves of the three unit c-FPD framework in Fig. 3 exhibit a linear relation typical of elastobrittle material. The inclination of the slope of the curve in Fig. 3 indicates the framework's rigidity, i.e. a structural ability to withstand deformation. A steeper slope means greater rigidity and more ability to resist deformation in the substance. When the lingual cusp of the framework pontic was loaded at an angle of $45^{\circ}$, the bearing capacity of the framework was $849 \mathrm{~N}$ and the displacement was $0.052 \mathrm{~mm}$. When the lingual cusp was loaded at $90^{\circ}$, the bearing capacity was $952 \mathrm{~N}$ and the displacement was $0.028 \mathrm{~mm}$. When the buccal cusp was loaded at $45^{\circ}$, the bearing capacity of the framework was $711 \mathrm{~N}$ and the displacement was $0.037 \mathrm{~mm}$. When loaded at $90^{\circ}$ on the buccal cusp, the bearing capacity of the framework was $965 \mathrm{~N}$ and the displacement was $0.026 \mathrm{~mm}$. That is, the bearing capacity and the rigidity were greatest when the loading was at $90^{\circ}$ angle, both for loading on the buccal and the lingual cusp. A higher rigidity of the framework could be observed when the buccal cusp was loaded than when the lingual cusp was loaded (Fig. 4). The difference in rigidity between loading on the buccal and the lingual cusps is smallest when a $90^{\circ}$ loading was performed and largest when a $60^{\circ}$ loading was performed (Fig. 4). Quadratic regressions through each five calculated results for each cusp results in two almost parallel curves and the differences between the curves range

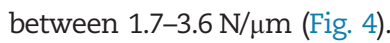

\subsection{Fracture pattern}

Fig. 5 presents the simulated fracture surfaces with loading on the buccal and lingual cusp at $45^{\circ}$ or $60^{\circ}$ angles. The fracture process could be followed step by step and revealed that the initial fracture was located in the occlusal area with the fracture in the gingival part identified as occurring later in the fracture process. When loading was on the buccal cusp, the fracture initiation site in the gingival embrasure of the framework occurred towards the buccal side of the framework. When loading was on the lingual cusp, the fracture initiation site in the gingival embrasure of the framework occurred towards the lingual side of the framework (Fig. 5). When loading was at a $60^{\circ}$ angle, the fracture initiation site in the gingival embrasure was located more towards the medial direction than when loading was at a $45^{\circ}$ angle (Fig. 5). With loading at a $60^{\circ}$ angle, a wider fracture surface, involving the area all the way to the edge of the sample, was observed; whereas when loading at a $45^{\circ}$ angle, a smaller area of fracture could be observed, leaving an unfractured area near the border of the framework. In Fig. 5 the crack tip, that is the edge of the crack, on the buccal side was higher, towards the occlusal surface, compared to its position on the lingual side when the buccal cusp was loaded. Loading at $45^{\circ}$, the appearance of the crack tip leaned more obliquely compared to when loaded at $60^{\circ}$, when the crack tip was more horizontally positioned (Fig. 5).

\section{Discussion}

In the oral cavity mechanical loading is seldom static but rather dynamic, for example, while chewing. Chewing in man is often performed in circular movements and the food between the teeth is crushed during grinding movements, meaning that the loading direction is often not fixed and can be in a variety of directions (Hiiemae et al., 1996). In the survey of the literature in the PubMed database, no studies were found which could show how loading at different loading positions and angles affects the fracture of c-FPD frameworks. Therefore, in the present study oblique loading on the pontic of a three-unit c-FPD framework was simulated using a 3D numerical analysis method.

In the present study the main fracture mechanism in the three-unit c-FPD framework could be identified as tensile stress failure and the crucial location for fracture was identified as being in the gingival embrasure. These findings are in agreement with those in previous numerical simulation analyses (Kelly 


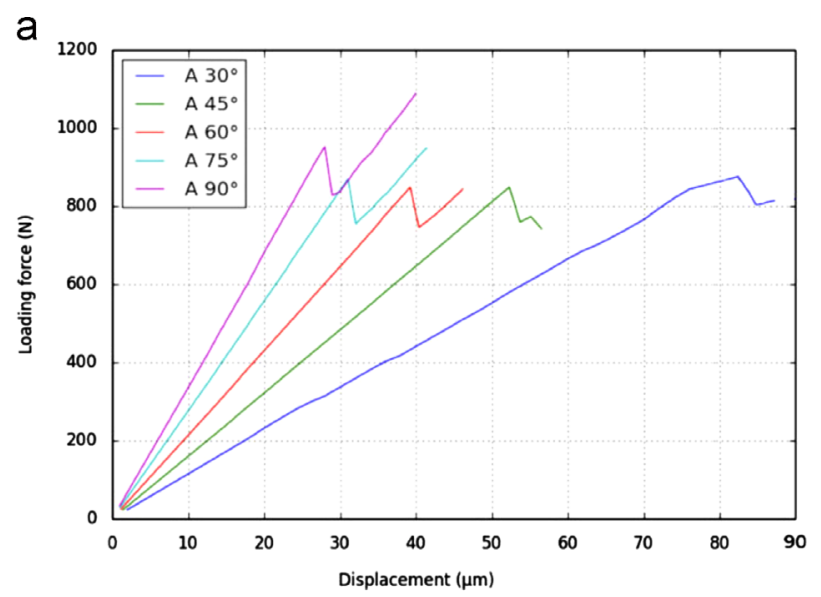

b

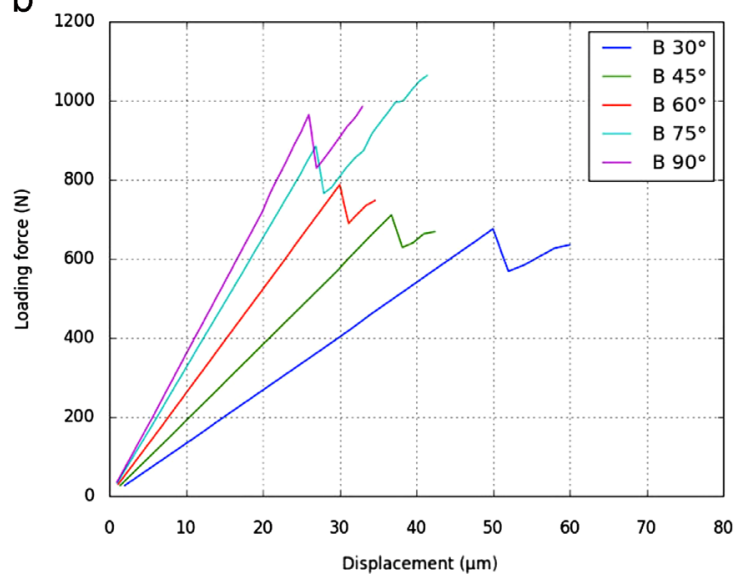

Fig. 3 - Loading-displacement curves of the three-unit c-FPD framework subjected to oblique loading with $30^{\circ}, 45^{\circ}, 60^{\circ}$, $75^{\circ}$ and $90^{\circ}$ angles. The framework loaded on the lingual cusp of the premolar shaped pontic denoted as A (Fig. 1a) and the buccal cusp denoted as B (Fig. 1b). Loading at $90^{\circ}$ angle, the highest bearing capacity and rigidity was exhibited both for loading on the buccal and lingual cusp. (a) For loading on the lingual cusp, a descending pattern of the rigidity of the framework could be observed from loading at $90^{\circ}$ to loading at $30^{\circ}$. The lowest bearing capacity was found for loading at $45^{\circ}$ and $60^{\circ}$. (b) For loading on the buccal cusp, a descending pattern of the bearing capacity and rigidity could be observed from loading at $90^{\circ}$ to loading at $30^{\circ}$. The lowest bearing capacity and rigidity of the framework was showed with loading at $30^{\circ}$ angle.

et al., 1995; Oh and Anusavice, 2002; Oh et al., 2002; Kou et al., 2007; Dittmer et al., 2009; Kou et al., 2011). However, one unique feature in the present 3D numerical simulation is the possibility it offers to follow the whole fracture process from beginning to end (Fig. 5). Once the initiation of the fracture was detected in the gingival embrasure the fracture propagation developed rapidly when the framework was subjected to a continued static loading. The fracture finally met the damaged area in the occlusal loading area and a complete picture of the total failure of the framework could be obtained (Figs. 2 and 5). Another
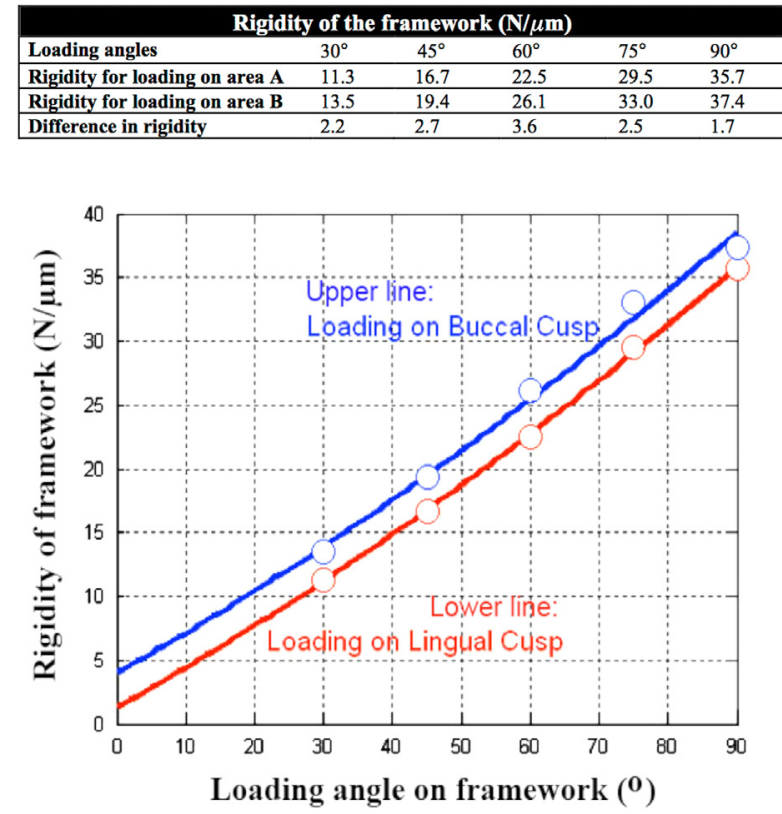

Fig. 4 - The rigidity of the three-unit c-FPD framework $(\mathrm{N} / \mu \mathrm{m})$ subjected to oblique loading on either the buccal or the lingual cusp at $30^{\circ}, 45^{\circ}, 60^{\circ}, 75^{\circ}$ and $90^{\circ}$. Quadratic regressions through each five calculated results for each cusp are shown. In the diagram the loading on the buccal cusp is shown in color blue and the loading on the lingual cusp in color red. (For interpretation of the references to color in this figure legend, the reader is referred to the web version of this article.)

interesting finding in the present simulation is that a clear correlation could be observed between the bearing capacity and rigidity of the three-unit c-FPD framework when it was subjected to oblique loading (Figs. 3 and 4).

\subsection{Validation of the numerical simulation}

Comparison the results obtained in the earlier 3D numerical simulation study (Kou et al., 2011) with a previous laboratory test (Sundh et al., 2005) reveals that the fracture patterns in the numerical study and the laboratory test were in agreement with each other. In both studies the fracture went between one of the connectors and diagonally through the loading point and no fracture could be observed in the abutments. Moreover, comparison between the two studies shows that the fracture pattern in the gingival part in the $3 \mathrm{D}$ numerical simulation (Kou et al., 2011) correlated well to the laboratory test (Sundh et al., 2005) and a compression curl could be seen in both the 3D numerical simulation and the laboratory test. The results in the previous 3D numerical study (Kou et al., 2011) were also compared with a previous fractographic analysis (Kou and Sjögren, 2010). The fracture initiation sites in the fractographic analysis were similar as in the previous 3D numerical modeling, the fracture mechanism was tensile failure and the fracture initiation was identified in the right gingival portion of the framework.

In addition, validation of the numerical code used in the present study was made by simulating a one-dimensional 

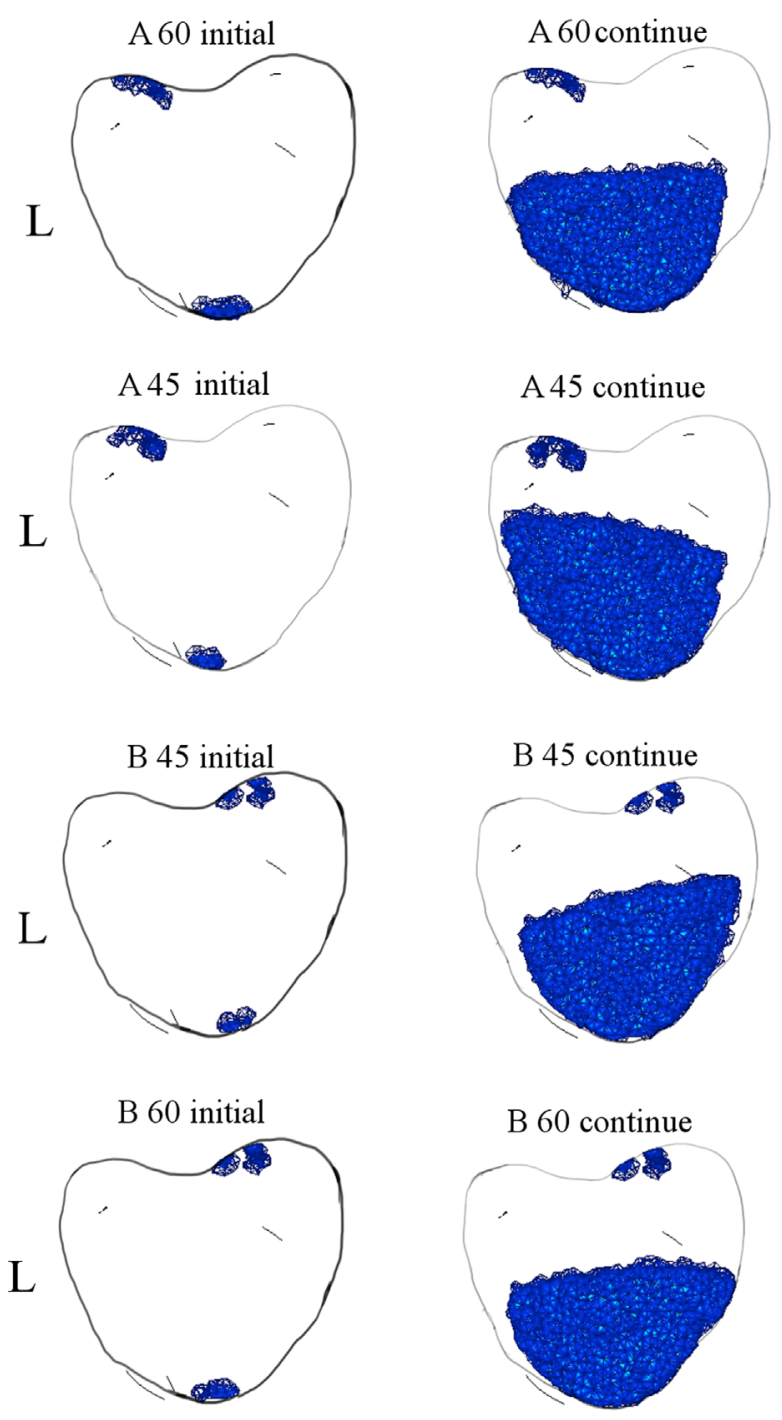

Fig. 5 - Images of simulated fracture surfaces during the fracture process. Loading on the lingual cusp at $45^{\circ}$ angle (A 45 ) and at $60^{\circ}$ angle (A 60). Loading on the buccal cusp at $45^{\circ}$ angle (B 45) and at $60^{\circ}$ angle (B 60). The figures present the initiation of the fracture process in the framework, denoted as 'initial', and the fracture process in progression, denoted as 'continue'. The color blue indicates the fractured areas. $\mathrm{L}$ denotes lingual direction. (For interpretation of the references to color in this figure legend, the reader is referred to the web version of this article.)

coal gas outburst based on a model considering seepage damage, strength discontinuity of mixed phase flow and air compression wave (Chen et al., 2014a). The results of the simulation were in accordance with the experimental results (Chen et al., 2014a). Moreover, Chen et al. (2014b) implemented a 3D model for gas and coal outburst and used the present numerical code to simulate the experiment carried out in the coal shockwave tube and the simulated results were approximately in agreement with the outburst experiment (Chen et al., 2014b).

\subsection{Simplification of the mechanical model}

When performing numerical simulation the mechanical models are often simplified (Tanimoto et al., 2002). A detailed calculation of the stress distribution and failure, taking all the factors in the real oral situation into consideration, is still not possible indicating the need for simplified models for handling the complexity. In clinical situations c-FPDs often consist of two layers; a core made of a reinforced ceramic material and a veneer of feldspar-based or glass ceramics (Miyazaki et al., 2013). Since the bearing material of c-FPDs restorations is mainly the core material, the outer veneer layer was not simulated in the present study. In addition, as in a previously performed numerical simulation study (Kou and Sjögren, 2011), a simplification in the abutments was made, since earlier laboratory studies (Sundh et al., 2005; Sundh and Sjögren, 2006; Kou and Sjögren, 2010) have shown that the fracture seldom occurs in the stainless steel abutments or in the retainer. Therefore a model with zirconia-based abutments with soft-layers at the base was simulated in the present study. The soft-layers in the base were to reduce the load on the zirconia abutments in order to avoid their fracture.

\subsection{Comparison between loading at $30^{\circ}, 40^{\circ}, 60^{\circ}, 75^{\circ}$ or $90^{\circ}$ angles}

In the present study loading of a c-FPD framework was simulated using loading angles that ranged from $30^{\circ}$ to $90^{\circ}$. The highest bearing capacity could be observed for the simulated framework loaded at a $90^{\circ}$ angle, i.e. perpendicular to the occlusal area of the pontic (Fig. 4). That is, when the framework was loaded only perpendicularly its survival could probably be prolonged. In addition, based on the findings in the present study a loading angle of $90^{\circ}$ resulted in high rigidity in the framework, whereas loading at an angle of $30^{\circ}$ resulted in low framework rigidity (Fig. 4). A lower loading angle indicated higher displacement of the framework, the result of which is that loading at a $30^{\circ}$ angle largely deforms the framework before it fractures, whereas loading at a $90^{\circ}$ angle deforms the framework less. Thus, in the present study it could be shown that the framework loaded at a $90^{\circ}$ angle towards the occlusal surface exhibited the highest bearing capacity and rigidity, whereas loading at a $30^{\circ}$ angle showed the lowest framework rigidity.

\subsection{Comparison between loading on the buccal or lingual cusp}

Loading on the buccal cusp of the pontic resulted in a greater rigidity in the framework compared to loading on the lingual cusp (Fig. 4). The anatomy of the simulated premolar-shaped pontic was similar to a real premolar, where the buccal cusp is often a bit sharper and larger while the lingual cusp is smaller and has a blunter appearance (Fig. 1c). Earlier clinical studies (Cavel et al., 1985; Eakle et al., 1986; Khers et al., 1990; Qian et al., 2013) suggest that cuspal anatomy plays a critical 
role in the fracture potential of cusps, and a higher fracture frequency is found in nonfunctional cusps than in functional cusps. One possible reason suggested is that the nonfuctional cusp is often smaller and may be more prone to fracture because of its size (Khers et al., 1990) and that occlusal force applied to these cusps will probably have a greater lateral component leading to cuspal fracture (Cavel et al., 1985) which could partly explain why the buccal cusp deforms less than the lingual cusp.

When loading at a $90^{\circ}$ angle similar level of bearing capacity could be observed in the framework, regardless of whether the lingual (A) or the buccal cusp (B) was loaded (Fig. 3). One possible reason for this could be that when vertical loading is applied the stress may be more evenly distributed throughout the FPD framework and therefore the outcome of the values might be more even for both framework rigidity and bearing capacity. A clear relationship between the load and displacement of various loading angles could be identified for loading on the buccal cusp, where a lower loading angle will cause lower bearing capacity and higher displacement. However, the relationship of the loading on the lingual cusp is more difficult to explain. The levels of the bearing capacity for loading at $60^{\circ}$ or $45^{\circ}$ exhibited similar values, but the bearing capacity for loading at $30^{\circ}$ was higher than loading at $60^{\circ}$, or $45^{\circ}$ angles. One possible explanation could be the anatomic surface irregularity of the lingual cusp of the simulated premolar (Fig. 1b).

\subsection{Fracture surface comparison with fractographic analysis}

The use of the present numerical simulation code allowed the fracture surface to be extracted, which as far as we know, has not been demonstrated before. In an earlier fractographic analysis of samples obtained from laboratory tests (Kou and Sjögren, 2010) it was demonstrated that static loading often causes high stress in the loading area and contact damage could be observed in the occlusal area (Fig. 6A). In this previous analysis, it was not possible to show when or why the contact damage occurred. According to the present numerical simulation, the occurrence of the fracture in the occlusal area of the loaded pontic could be determined as the initial fracture in the framework (Fig. 5). In addition, the fracture in this area seems to be due to the concentration of the compressive stress but the fracture developed slowly compared to the fracture in the gingival part. These findings are in agreement with the fracture propagation process received from the numerical simulation performed earlier (Kou et al., 2011) of a ceramic framework with a geometric design similar to that in the current study.

Analyzing the simulated fracture surface shows that the location of the fracture initiation sites seems to vary when the ceramic framework is loaded on different cusps and also at various angles (Fig. 5). When the loading is at the buccal cusp the location of the fracture initiation site occurs in the middle of the gingival part and deviates a little in the buccal direction (Fig. 5). When loading is on the lingual cusp the fracture initiation site is located in the middle of the gingival part and deviates a little in the lingual direction (Fig. 5). This finding can partly explain the fracture pattern from an earlier laboratory study (Kou and Sjögren, 2010). In the earlier fractographic analysis (Kou and Sjögren, 2010) the loading was performed perpendicularly both on the buccal and lingual cusps on a pontic-shaped premolar with a geometric design similar to that in the present numerical simulated model. The fracture initiation was found on the gingival embrasure towards the buccal side of the framework (Fig. 6B). Based on the present simulation results the explanation could be that the loading on the buccal cusp might be more prominent than the loading on the lingual cusp. With a $90^{\circ}$ loading, the location of the fracture initiation site might occur vertically towards the loading area, whereas with a more oblique loading, such as loading at $30^{\circ}$, it could move towards the buccal or lingual directions (Fig. 5). The fracture surface also seems to differ in appearance with different loading conditions (Fig. 5). With a $45^{\circ}$ loading on the buccal cusp, it could be observed that the fracture surface appeared to be more oblique and the crack tip of the fracture surface leaned more in the buccal direction, and vice versa for the lingual cusp. The width of the fracture
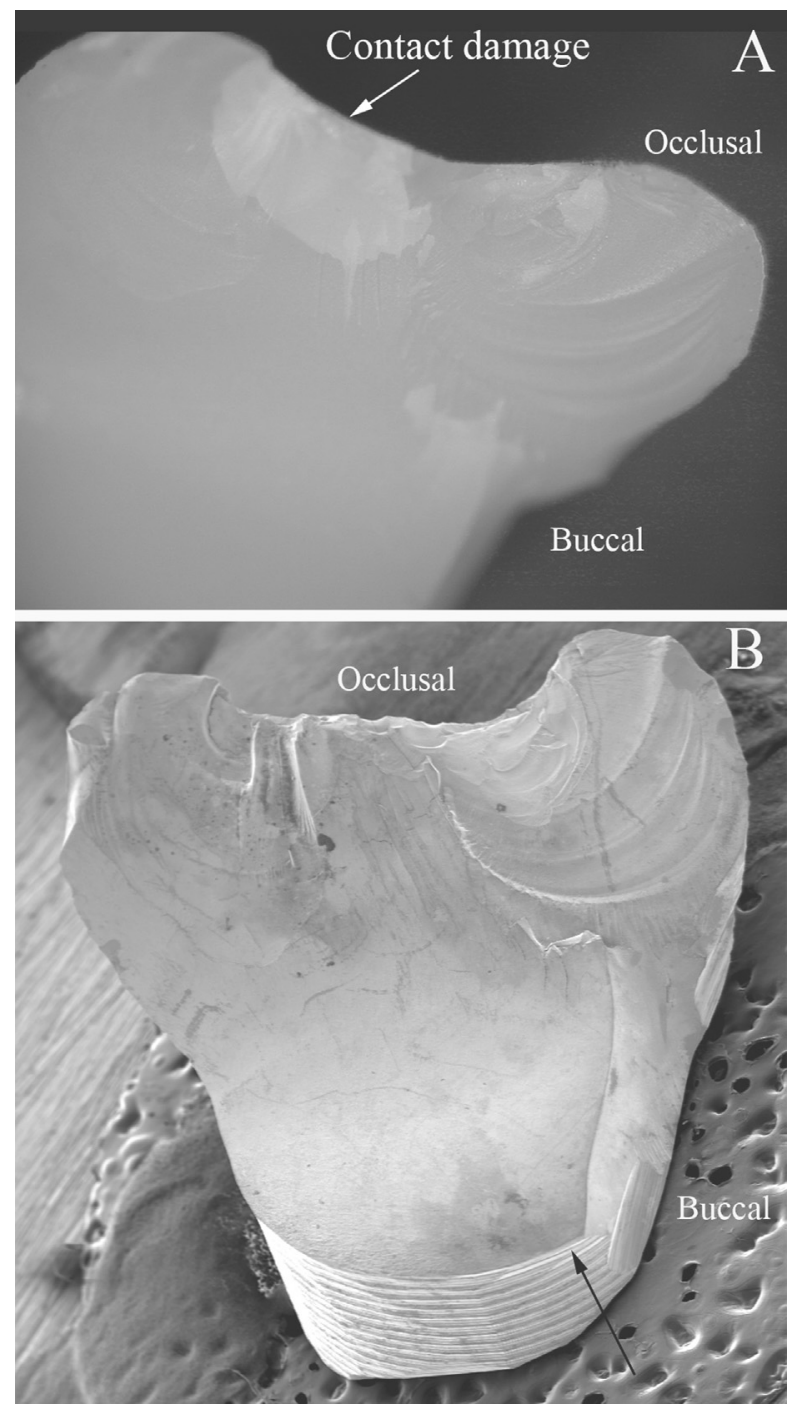

Fig. 6 - Images of selected fractured surface areas from an earlier performed fractographic analysis (Kou and Sjögren, 2010). (A) The occlusal surface shows contact damage and the white colored irregularities are probably cracks within the thickness of the Y-TZP. (B) Scanning electron microscope (SEM) image of a fractured surface at $12 \times$ magnification and the fracture initiation is marked with a black arrow. 
surface area also varies: with a loading at $60^{\circ}$ the crack tip covered the whole way towards the edge of the fracture surface, whereas with a loading at $45^{\circ}$ the coverage of the fracture surface has a narrower appearance (Fig. 5).

Stress concentration seems to be the main reason for the initiation of fracture in ceramic frameworks and stress concentration should therefore be avoided as much as possible. If defects and voids in the ceramic material could be avoided, a higher survival rate of the c-FPD could probably be achieved. It is important therefore to identify the localization of the stress concentration areas when designing c-FPD frameworks and individual analysis of stress distribution in c-FPDs would probably optimize the design of the restorations and increase their survival rate.

In summary, using the present 3D numerical simulation the whole fracture propagation process could be followed step by step from the beginning to the total failure of the ceramic framework (Figs. 2 and 5). Compared to conventional fractographic analysis, the present simulation could provide complementary information about the fracture process. Thus, the 3D numerical simulation applied here could serve as a complement to conventional fractographic analysis in gaining overall knowledge about fractures in this type of ceramic restoration. However, the 3D numerical simulation in the present study could not exactly simulate the real oral situation and, therefore, simplified models were used. For example, in the real oral situation the abutments consist of natural teeth with surrounding alveolar bone and periodontium, whereas in the present study a model with zirconia-based abutments was simulated.

\subsection{Clinical implication}

The present study indicates that oblique loading has a major impact on the fracture of ceramic frameworks. The survival time for c-FPD frameworks would probably increase if their design could be better adapted to the patient's chewing pattern.

More knowledge is still needed to clarify in what way various chewing patterns affect loading and fracture modes of c-FPDs and how the present numerical code could be adapted to suggest a specific design of individual c-FPDs, e.g. the relationship between loading direction and cuspal anatomy.

\section{Conclusion}

The numerical simulation performed here made it possible to follow the progress of fracture in a three-unit c-FPD subjected to oblique loading and the following conclusions could be drawn:

i. The cause of fracture in the c-FPD framework simulated in the present study was mainly tensile stress.

ii. The fracture of the c-FPD framework, which started in the occlusal area, was due to compression; however, the crucial fracture location that caused catastrophic failure of the framework occurred in the gingival part of the framework.

iii. The load capacity and rigidity of the simulated framework varied with the loading position and direction. The c-FPD framework can bear the greatest loading and cannot be easily deformed when the loading direction is perpendicular to the occlusal surface. Therefore, loading in the vertical direction is preferable with regard to the durability of all-ceramic frameworks.

iv. The crack tip of the fracture surface was influenced by the loading location and direction.

\section{Acknowledgments}

The authors would like to thank the Faculty of Medicine, Umeå University, Umeå, Sweden and the Swedish Dental Association for financial support. We also acknowledge Cad. esthetics $A B$, Skellefteå, Sweden for providing data of the mechanical properties of the ceramic framework.

Notes: Reprinting permission for Figs. 1 and 6 are received from the publisher (Wiley Periodicals Inc.) with license numbers 3531251107241 and 3526430223722.

\section{R E F E R E N C E S}

Cavel, W.T., Kelsey, W.P., Blankenau, R.J., 1985. An in vivo study of cuspal fracture. J. Prosthet. Dent. 53, 38-42.

Cehreli, M.C., Kokat, A.M., Ozpay, C., Karasoy, D., Akca, K., 2011. A randomized controlled clinical trial of feldspathic versus glass-infiltrated alumina all-ceramic crowns: a 3-year followup. Int. J. Prosthodont. 24, 77-84.

Chai, J., Chu, F.C., Chow, T.W., Liang, B.M., 2007. Chemical solubility and flexural strength of zirconia-based ceramics. Int. J. Prosthodont. 20, 587-595.

Chen, L., Qiao, J., Ding, Y., 2014a. A model of one-dimensional coal gas outburst wave (in Chinese). Sci. Sin-Phys. Mech. Astron. 44, 68-78.

Chen, L., Qiao, J., Ding, Y., 2014b. Three-dimensional model for estimating coal and gas outburst (in Chinese). Mechan. Eng. 36, 168-171.

Depprich, R., Zipprich, H., Ommerborn, M., Naujoks, C., Wiesmann, H.P., Kiattavorncharoen, S., Lauer, H.C., Meyer, U., Hübler, N.R., 2008. Handschel. Osseointegration of zirconia implants compared with titanium: an in vivo study. Head Face Med. 4, 1-8.

Dittmer, M.P., Kohorst, P., Borchers, L., Stiesch-Scholz, M., 2009. Finite element analysis of a four-unit all-ceramic fixed partial denture. Acta Biomater. 5, 1349-1355.

Eakle, W.S., Maxwell, E.H., Braly, B.V., 1986. Fractures of posterior teeth in adults. J. Am. Dent. Assoc. 112, 215-218.

Ghazal, M., Steiner, M., Kern, M., 2008. Wear resistance of artificial denture teeth. Int. J. Prosthodont. 21, 166-168.

Guazzato, M., Albakry, M., Ringer, S.P., Swain, M.V., 2004. Strength, fracture toughness and microstructure of a selection of allceramic materials. Part II: zirconia-based dental ceramics. Dent. Mater. 20, 449-456.

Guazzato, M., Quach, L., Albakry, M., Swain, M.V., 2005. Influence of surface and heat treatments on the flexural strength of Y-TZP dental ceramic. J. Dent. 33, 9-18.

Hiiemae, K., Heath, M.R., Heath, G., Kazazoglu, E., Murray, J., Sapper, D., et al., 1996. Natural bites, food consistency and feeding behaviour in man. Arch. Oral. Biol. 41, 175-189.

Holberg, C., Winterhalder, P., Wichelhaus, A., Hickel, R., Huth, K., 2013. Fracture risk of lithium-disilicate ceramic inlays: a finite element analysis. Dent. Mater. 29, 1244-1250. 
Håff, A., Löf, H., Gunne, J., Sjögren, G., 2015. A retrospective evaluation of zirconia-fixed partial dentures in general practices: an up to 13-year study. Dent. Mater. 31, 162-170.

Kelly, J.R., Tesk, J.A., Sorensen, J.A., 1995. Failure of all-ceramic fixed partial dentures in vitro and in vivo: analysis and modeling. J. Dent. Res. 74, 1253-1258.

Khers, S.C., Carpenter, C.W., Vetter, J.D., Staley, R.N., 1990. Anatomy of cusps of posterior teeth and their fracture potential. J. Prosthet. Dent. 64, 139-147.

Kou, W., Kou, S., Liu, H., Sjögren, G., 2007. Numerical modeling of the fracture process in a three-unit all-ceramic fixed partial denture. Dent. Mater. 23, 1042-1049.

Kou, W., Sjögren, G., 2010. Fracture behaviour of zirconia FPDs substructures. J. Oral Rehabil. 37, 292-299.

Kou, W., Li, D., Qiao, J., Chen, L., Ding, Y., Sjögren, G., 2011. A 3D numerical simulation of stress distribution and fracture process in a zirconia-based FPD framework. J. Biomed. Mater. Res.: B Appl. Biomater. 96, 376-385.

Lee, J.S., Lim, Y.J., 2013. Three-dimensional numerical simulation of stress induced by different lengths of osseointegrated implants in the anterior maxilla. Comput. Methods Biomech. Biomed. Eng. 16, 1143-1149.

Liu, H.Y., 2004. Numerical modeling of the rock fragmentation process by mechanical tools (Doctoral thesis). Luleå University of Technology, Luleå, Sweden.

Miyazaki, T., Nakamura, T., Matsumura, H., Ban, S., Kobayashi, T., 2013. Current status of zirconia restoration. J. Prosthodont. Res. 57, 236-261.

Munz, D., Fett, T., 1999. In: Ceramics: Mechanical Properties, failure Behaviour, Materials Selection. Springer-Verlag, Berlin.
Oh, W.S., Anusavice, K.J., 2002. Effect of connector design on the fracture resistance of all-ceramic fixed partial dentures. J. Prosthet. Dent. 87, 536-542.

Oh, W., Gotzen, N., Anusavice, K.J., 2002. Influence of connector design on fracture probability of ceramic fixed-partial dentures. J. Dent. Res. 81, 623-627.

Qian, Y., Zhou, X., Yang, J., 2013. Correlation between cuspal inclination and tooth cracked syndrome: a three-dimensional reconstruction measurement and finite element analysis. Dent. Traumatol. 29, 226-233.

Papanicolaou, G.C., Kouveliotis, G., Nikolopoulou, F., Papaefthymiou, K.P., Bairami, V., Portan, D.V., 2015. Thermal shock cycling effect on the compressive behaviour of human teeth. J. Biomech. 48, 681-686.

Sorensen, J.A., Kang, S.K., Torres, T.J., Knode, H., 1998. In-Ceram fixed partial dentures: three-year clinical trial results. J. Calif. Dent. Assoc. 26, 207-214.

Sundh, A., Molin, M., Sjögren, G., 2005. Fracture resistance of yttrium oxide partially-stabilized zirconia all-ceramic bridges after veneering and mechanical fatigue testing. Dent. Mater. 21, 476-482.

Sundh, A., Sjögren, G., 2006. Fracture resistance of all-ceramic zirconia bridges with differing phase stabilizers and quality of sintering. Dent. Mater. 22, 778-784.

Tanimoto, Y., Nishiwaki, T., Nishiyama, N., Nemoto, K., Maekawa, Z., 2002. A simplified numerical simulation method of bending properties for glass fiber cloth reinforced denture base resin. Dent. Mater. J. 21, 105-117.

Weibull, W., 1951. A statistical distribution function of wide applicability. J. Appl. Mech. 18, 293-297. 